\title{
pesquisa
}

\section{EMCEFALOCELE OCCIPITAL: REVISÃO BIBLIOGRÁFICA COM UM RELATO DE CASO}

Mozzer EB*

Universidade do Oeste de Santa Catarina https://orcid.org/0000-0002-9122-8910

Bonatto KZB ${ }^{\dagger}$

Universidade do Oeste de Santa Catarina https://orcid.org/0000-0001-5102-991X

Debiasi $\mathbf{M M}^{\ddagger}$

Universidade do Oeste de Santa Catarina https://orcid.org/0000-0003-4944-9645

D’Agostini FM ${ }^{\S}$

Universidade do Oeste de Santa Catarina https://orcid.org/0000-0002-1115-6153

Resumo: Os defeitos do sistema nervoso acometem 1 a 10:1.000 dos nascidos vivos e são as malformações mais comuns detectadas nos fetos, sendo a maioria delas diagnosticada pelo ultrassom do segundo trimestre da gestação. A encefalocele, que consiste na herniação do cérebro e/ou das meninges através de um defeito na calota craniana, está entre os quatro principais defeitos do fechamento do tubo neural durante o desenvolvimento embrionário. Assim, o objetivo deste estudo foi realizar uma revisão de literatura sobre encefalocele occipital e apresentar um relato de caso de natimorto diagnosticado com essa malformação. Para a revisão bibliográfica foram consultadas

\footnotetext{
* Graduanda em Medicina pela Universidade do Oeste de Santa Catarina; manu_mozzer2@hotmail.com

† Graduanda em Medicina pela Universidade do Oeste de Santa Catarina; karenbonatto@gmail.com

¥ Mestre em Ciência e Biotecnologia pela Universidade do Oeste de Santa Catarina; Especialista em Biologia Celular e Tecidual pela Universidade Federal do Paraná; marcelina.debiasi@unoesc.edu.br

§ Doutora em Zoologia pela Pontifícia Universidade Católica do Rio Grande do Sul; Mestre em Biociências pela Pontifícia Universidade Católica do Rio Grande do Sul; fernanda.dagostini@unoesc.edu.br
} 
bases de dados nacionais e internacionais referentes ao período de dezembro de 2016 a agosto de 2018; já no relato de caso fez-se a descrição de exames clínicos e imaginológicos, bem como a evolução clínica da paciente. Parte das anomalias relacionadas aos defeitos de fechamento do tubo neural, como a encefalocele, não apresenta uma causa específica conhecida, dessa forma, faz-se necessária a divulgação de estudos explorando de forma detalhada esse tema.

Palavras-chave: Malformações. Tubo neural. Herniação do cérebro.

\title{
Occipital encefalocele: bibliographic review with a case report
}

\begin{abstract}
The defects of the nervous system affect 1 to 10:1000 of the live births and are the most common malformations detected in the fetuses, most of them diagnosed by the ultrasound of the second trimester of gestation. The encephalocele, which consists of herniated brain and/or meninges through a defect in the skull cap, is among the four major defects of neural tube closure during embryonic development. Therefore, the objective of this study was to carry out a review of literature on occipital encephalocele and present a case report of a stillborn child diagnosed with this malformation. For the bibliographic review, national and international databases referring to the period from December 2016 to August 2018 were consulted, as well as a description of clinical and imaging exams as well as the clinical evolution of the patient. Some of the anomalies related to neural tube closure defects, such as the encephalocele, do not present a specific known cause, so it is necessary to disseminate studies exploring this topic in detail.
\end{abstract}

Keywords: Malformations. Neural tube. Herniation of the brain.

Recebido em 25 de abril de 2019

Aceito em 22 de maio de 2019

\section{INTRODUÇÃO}

As malformações congênitas são distúrbios do desenvolvimento que surgem no período embrionário e podem ser de origem estrutural, funcional ou metabólica, causando anomalias físicas ou mentais.' A incidência desses distúrbios é de aproximadamente $5 \%$ dos nascidos vivos na América Latina, ${ }^{2}$ destacando-se a encefalocele-que consiste na herniação do cérebro e das meninges em decorrência de um defeito durante o desenvolvimento 
na calota craniana - entre os quatro principais defeitos do fechamento do tubo neural. ${ }^{3}$ Outro dado importante é que a maior parte dessas anomalias permanece sem uma causa específica. Todavia, alguns fatores de risco já foram elencados, como: medicamentos, tabagismo, alcoolismo, uso de drogas ilícitas, exposições ambientais ou ocupacionais, diabetes mellitus gestacional, disfunção da tireoide e infecções congênitas. ${ }^{4}$

Estudos mostram que os defeitos do sistema nervoso central são as malformações mais comuns detectadas nos fetos, sendo a maioria delas diagnosticada pelo ultrassom do segundo trimestre (entre 20 e 24 semanas de gestação). ${ }^{4}$ Além disso, observa-se que mais de $70 \%$ dos casos evolvem para o óbito após o nascimento.' Nesse contexto, o presente artigo teve por finalidades relatar um caso explorando variáveis que possam ser úteis no manejo clínico da referida patologia e, também, estimular outros profissionais da Área das Ciências da Vida e Saúde a buscarem conhecimento mais detalhado a respeito desse tema.

\section{METODOLOGIA}

Este artigo foi elaborado com base em revisão de literatura a respeito das malformações do sistema nervoso central, destacando-se a encefalocele occipital. A revisão foi realizada nas bases de dados Scielo, Google Acadêmico e PubMed referente ao período de dezembro de 2016 a agosto de 2018. O relato de caso de encefalocele occipital foi descrito referente a um natimorto na região Meio-Oeste de Santa Catarina, respeitando a Resolução CNS n. 466/2012, com aprovação do comitê de ética em pesquisa com seres humanos, Parecer n. 2.560.551/2018.

\section{REVISÃO DE LITERATURA}

\subsection{EPIDEMIOLOGIA}

No Brasil, as malformações congênitas constituem a segunda causa de mortalidade infantil, sendo que os defeitos congênitos do sistema nervoso central (SNC) acometem 1 a 10:1.000 dos nascidos vivos. Essa estimativa pode variar de acordo com a sazonalidade, 
o país e os grupos étnicos ou quando observada em serviços de diagnóstico pré-natal e de neonatologia. ${ }^{5}$

No estudo Malformações do sistema nervoso central e malformações associadas diagnosticadas pela ultrassonografia obstétrica, os autores ${ }^{5}$ concluíram que os defeitos congênitos do SNC estavam presentes sem outras malformações associadas em 65,78\%, com a distribuição: hidrocefalia (37,5\%), mielomeningocele (15\%), encefalocele (12,5\%), agenesia de corpo caloso (12,5\%), anencefalia (12,5\%), holoprosencefalia (7,5\%), Dandy-Walker $(7,5 \%)$, Arnold-Chiari (5,0\%), hidranencefalia (5,0\%), meningocele $(5,0 \%)$ e cisto aracnoideo $(2,5 \%)$. A encefalocele foi a terceira anomalia congênita mais frequentemente associada tanto à mielomeningocele quanto à agenesia do corpo caloso, bem como à hidrocefalia. ${ }^{5}$

\subsection{DEFINIÇÃO E FISIOPATOLOGIA}

Encefaloceles são defeitos no tubo neural, associados a falhas no crânio, que podem levarà herniação das meningese do tecido cerebral (encefalocele ou meningoencefalocele) ou apenas das meninges (meningocele craniana). ${ }^{6}$ Essa malformação se deve a um erro na embriogênese durante a fusão do neurocranium cartilaginoso, do neurocranium membranoso ou do viscerocrânio, sendo que os casos mais graves ocorrem antes do $26^{\circ}$ dia de gestação, momento em que há o fechamento do neuroporo. ${ }^{8}$

Conforme o local acometido, a deformidade é classificada em: encefalocele occipital (ocorre em $75 \%$ dos casos), sincipital (frontoetmoidal, interfrontal e associada a fissuras craniofaciais), parietal ou basal (intrasfenoidal, transfenoidal, transetmoidal, esfenoetmoidal, fronto-esfenoidal ou esfeno-orbital). ${ }^{7}$ É importante saber a localização, pois pode haver modificação tanto no tratamento quanto no prognóstico do paciente, dependendo da classificação em que ele se encaixa. ${ }^{6}$

\subsection{ETIOLOGIA E FATORES DE RISCO}

As causas do defeito podem ser múltiplas, incluindo fatores genéticos e ambientais. Medicamentos (como ácido valproico, dipirona sódica, naproxeno sódico, ácido 
acetilsalicílico e vários analgésicos, anti-inflamatórios e antibióticos), condições infecciosas (vias aéreas superiores e inferiores, sistema geniturinário e TORCH - toxoplasmose, rubéola, citomegalovírus e herpes simples), irradiação, excesso de vitamina A, falta de vitamina B12, escassez de ácido fólico e suplementação mineral, administração de insulina, salicilatos, hipertermia e uso de álcool, tabaco ou drogas ilícitas são considerados fatores de risco. ${ }^{8}$

Indivíduos com histórico familiar de defeitos do tubo neural (espinha bífida ou anencefalia) podem ser mais suscetíveis a desenvolver uma encefalocele, porém um segundo caso não foi observado em nenhuma família nos estudos. ${ }^{9}$ Autores ${ }^{6}$ relatam que não há correlação desses defeitos com fatores ambientais, entretanto a encefalocele pode ocorrer como parte de mais de 30 síndromes diferentes (Mechel, Fraser, Roberts, Walter-Warburg).

A grande maioria dos defeitos abertos do tubo neural (DATN) está relacionada a causas multifatoriais. Entretanto, há diversos estudos que comprovam a importância da reposição gestacional de ácido fólico para as fases do desenvolvimento embrionário, visto que essa vitamina do complexo B participa de diversos processos em nível celular. ${ }^{10}$

Diante disso, o Ministério da Saúde preconiza a administração preventiva de ácido fólico no período pré-gestacional na dose de $5 \mathrm{mg}$ ao dia, 60 a 90 dias antes da concepção. Essa recomendação independe do risco e é repassada a todas as mulheres que pretendem engravidar." Contudo, deve-se considerar que mesmo havendo a suplementação correta dessa vitamina, ela não será capaz de prevenir plenamente a ocorrência de DATN. ${ }^{10}$

\subsection{DIAGNÓSTICO E PROGNÓSTICO}

O diagnóstico da encefalocele é realizado através do exame de ultrassom pré-natal, que evidencia a descontinuidade do osso do crânio. A imagem vista no exame pode se apresentar anecogênica ou de conteúdo denso; no primeiro caso, o prolapso apresenta apenas líquido em seu conteúdo, enquanto no segundo, há também massa encefálica em seu interior. ${ }^{12}$

Após o nascimento da criança pode-se realizar também o diagnóstico clínico através do exame físico que evidencia o prolapso, seguido de exames complementares, como radiografia de crânio, tomografia computadorizada e ressonância nuclear magnética. Entretanto, o diagnóstico pré-natal precoce é de suma importância para determinar o prognóstico e promover uma adequada investigação genética. ${ }^{12}$ 
Com relação ao prognóstico, este depende do conteúdo, da dimensão, da localização do saco herniário e de malformações associadas. ${ }^{12}$ Além disso, por evitar trauma e contaminação do tecido cerebral, acredita-se que a cesariana pode melhorar a evolução do caso. ${ }^{13}$ Ainda, estudos mostram que $16 \%$ das encefaloceles occipitais são maiores que $20 \mathrm{~cm}, 12 \%$ têm de 10 a $15 \mathrm{~cm}, 30 \%$, entre 5 e $10 \mathrm{~cm}$, e $28 \%$ são menores que $5 \mathrm{~cm}$, e que, independentemente do tamanho, a microcefalia está presente em 0-24\% dos casos, e a hidrocefalia em 20-65\% dos casos. ${ }^{8}$ Ocorrem, também, anomalias associadas (renal ou cardíaca) em aproximadamente $30 \%$ das crianças com encefalocele. ${ }^{9}$

De acordo com dados do Programa de Defeitos Congênitos de Atlanta Metropolitana, $76 \%$ das mortes de crianças comencefalocele ocorrem no primeiro dia de vida, e a probabilidade de sobrevivência de um ano é de $70,8 \%$. Os fatores associados ao aumento da mortalidade foram baixo peso ao nascer (< $2500 \mathrm{~g}$ ), presença de defeitos múltiplos e raça negra. ${ }^{14}$

\subsection{CONDUTA}

No que diz respeito à encefalocele, a literatura traz que optar pela cesariana como conduta pode melhorar o prognóstico do paciente por evitar contaminação ou trauma do tecido cerebral. Já nas situações em que há associação com microcefalia ou alguma patologia incompatível com a vida há indicação de conduta obstétrica conservadora. ${ }^{13}$

Nos casos em que ocorre óbito fetal, 75 a $90 \%$ das gestantes acabam evoluindo para parto espontâneo em duas semanas, porém, em razão de todo o envolvimento emocional que essa espera traz, poucas pacientes aceitam aguardar passivamente 0 evento, solicitando ao médico uma conduta ativa de intervenção. ${ }^{15}$

\section{RELATO DO CASO E DISCUSSÃO}

Gestante TCF, 27 anos de idade, residente na região Meio-Oeste de Santa Catarina, fez uso de ácido fólico $5 \mathrm{mg}$ três meses antes da concepção, mantendo-o no desenvolvimento da gestação. Gravidez única, desejada, controlada e sem antecedentes patológicos. O exame de sexagem fetal revelou um feto do sexo feminino. Por volta da 
$21^{a}$ semana de gestação evidenciou-se, por meio do exame de ultrassonografia (USG), crescimento fetal abaixo da média $(26,7-27,8 \mathrm{~cm})$ para a idade gestacional, presença de encefalocele occipital de moderado/acentuado volume, osso nasal hipoplásico, impressão de comunicação intraventricular (CIV) perimembranosa e ossos longos com tamanho abaixo da média (fêmur entre 3,1 e 3,4 mm; tíbia entre 2,8 e 3,0 mm; fíbula entre 2,7 e 2,9 mm; úmero entre 3 e 3,3 mm; rádio entre 2,5 e 2,7 mm; ulna entre 2,8 e 3,1 mm; pé entre 3,1 e $3,4 \mathrm{~mm}$ ) para a idade gestacional (Figuras 1 e 2).

Com 24 semanas e quatro dias a gestante foi submetida ao exame de amniocentese genética, que resultou no cariótipo 46, XX, não revelando nenhuma anormalidade cromossômica numérica ou estrutural. Decorrida uma semana da amniocentese, a gestante notou ausência de movimentação fetal, o que a fez procurar auxilio de seu médico. Este, após confirmar a ocorrência do óbito fetal através do USG, tentou induzir o trabalho de parto por meio de medicações, mas, sem sucesso, teve de recorrer à via de parto cesáreo. As justificativas atestadas como causa da morte foram: anóxia intrauterina; encefalocele occipital; malformações do sistema nervoso central e cardíaco; e polidrâmnio.

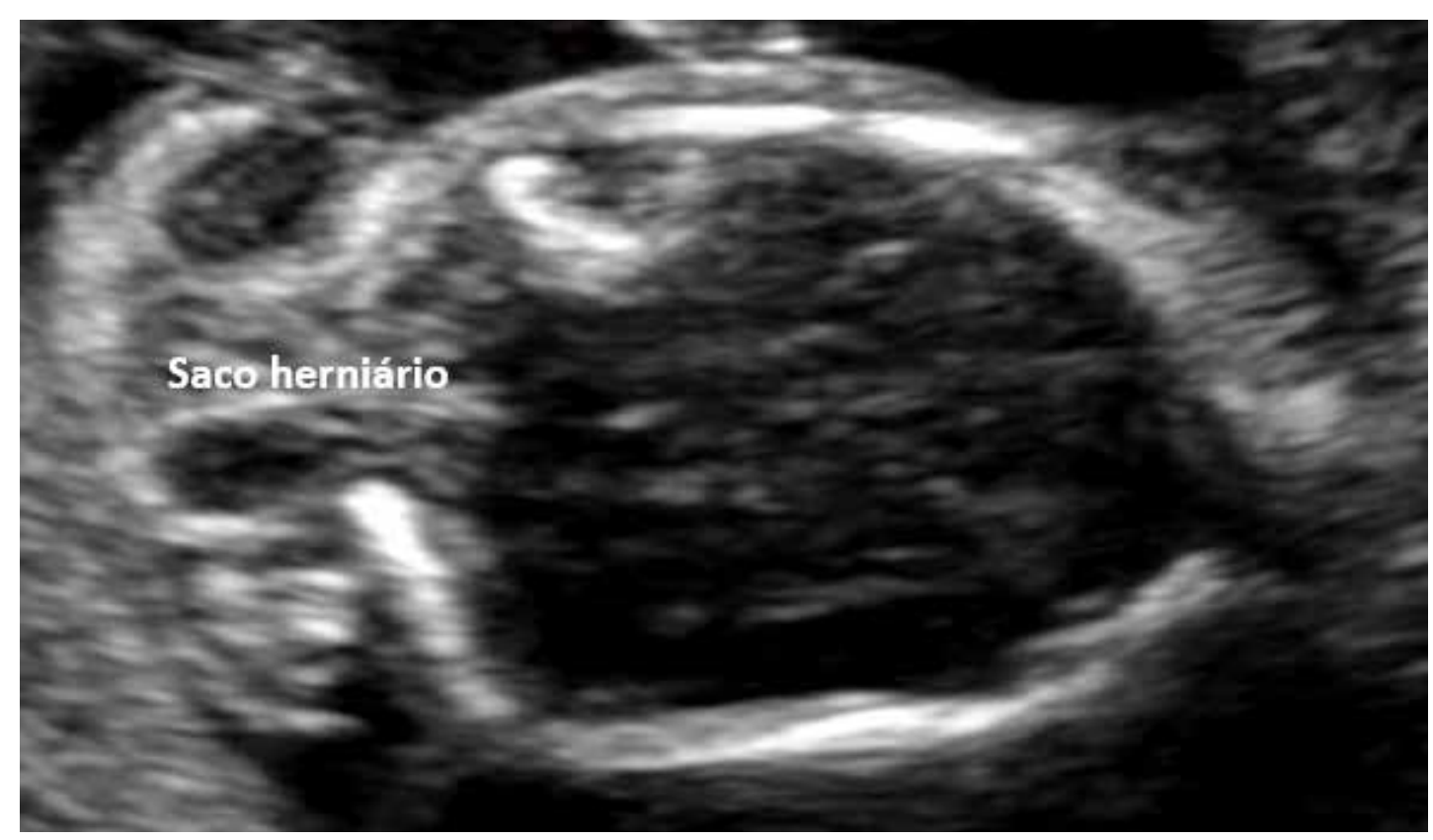

Figura 1 - Ultrassonografia evidenciando calota craniana com contornos regulares e reentrância ao nível dos ossos parietais

Nota: Observa-se defeito de fechamento da calota craniana na sua porção occipital, com a formação de saco herniário contendo líquor e tecido encefálicos, compatível com encefalocele. O saco herniário mede $39 \times 33 \times 40 \mathrm{~mm}$ (volume de $28,2 \mathrm{~cm}^{3}$ ). O defeito ósseo mede $10 \mathrm{~mm}$ (abertura ampla, região posterior). 


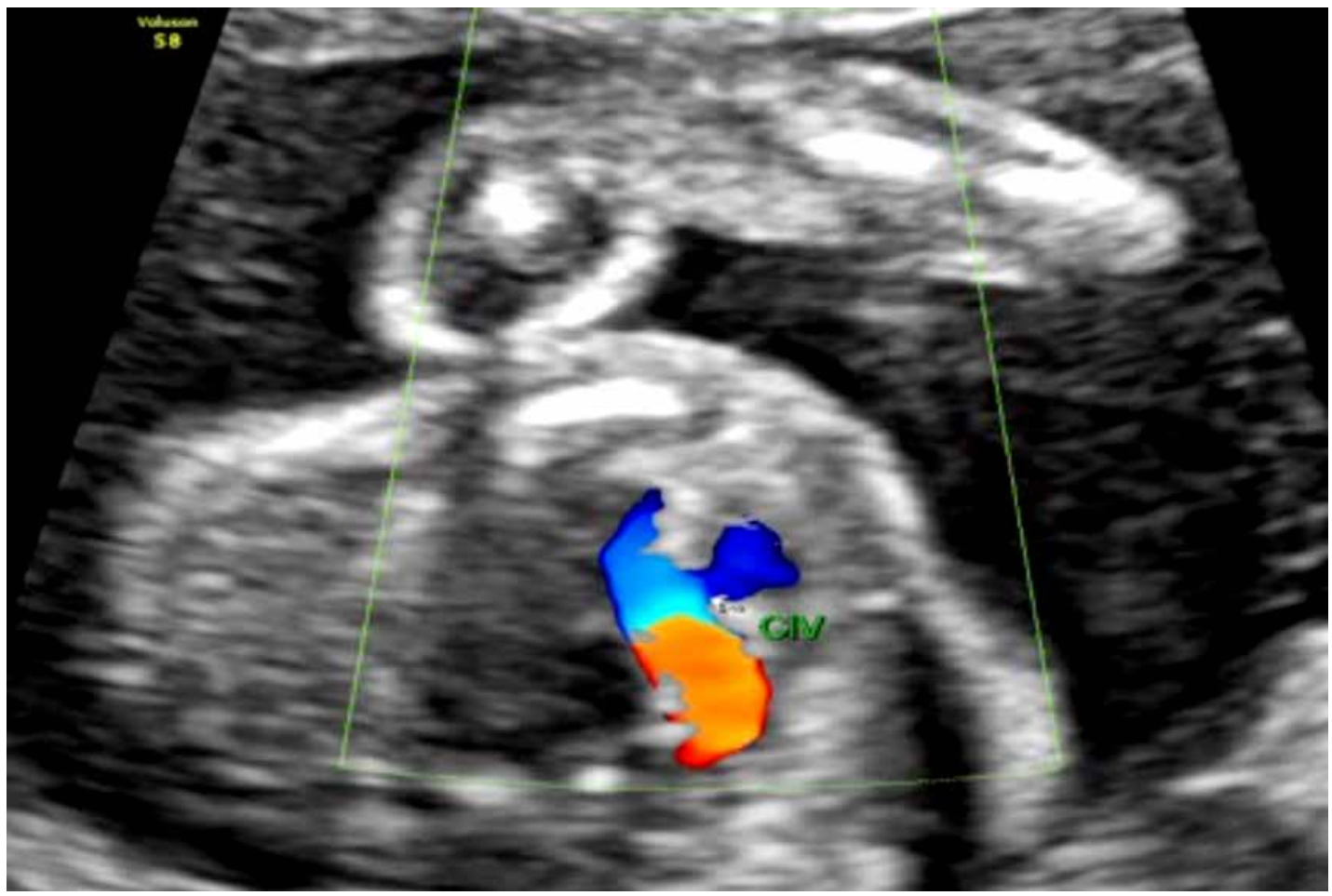

Figura 2 - Coração com pequena solução de continuidade, medindo 1,4 mm, na porção perimembranosa do septo interventricular (CIV)

Apesar de o mecanismo pelo qual o ácido fólico previne os defeitos abertos do tubo neural (DATN) ainda não estar completamente esclarecido, a necessidade do seu uso como prevenção já é consenso na literatura. Inclusive, sabe-se que baixo consumo na dieta (por meio de reduzida ingesta de feijão, vegetais verdes, frutas cítricas, etc.), distúrbios gênicos, tabagismo, uso crônico de contraceptivos hormonais orais, anticonvulsivantes e diabetes são exemplos de fatores relacionados à queda nos níveis séricos desse micronutriente, necessitando de reposição periconcepcional. ${ }^{12}$ Todavia, a gestante do presente relato, além de não apresentar em sua história nenhum desses fatores que pudessem depletar os níveis de folato, ainda realizou de forma adequada a reposição indicada no período pré-gestacional e gestacional, afastando esses elementos como possíveis causadores da encefalocele e deixando uma lacuna com relação à etiologia dessa patologia.

O exame imaginológico de escolha para diagnóstico das encefaloceles é a Ultrassonografia pré-natal demonstrando descontinuidade do osso do crânio. ${ }^{12}$ No caso da paciente, o USG evidenciou, também, osso nasal hipoplásico, impressão de 
comunicação interventricular (CIV) perimembranosa e ossos longos com tamanho abaixo da média para a idade gestacional. Além disso, havia crescimento fetal abaixo da média e presença de encefalocele occipital de moderado/acentuado volume. As encefaloceles occipitais são mais comuns nos fetos do sexo feminino do que nos do sexo masculino,' o que condiz com o caso da paciente.

Quanto ao tratamento desse DATN, em geral tem-se o acompanhamento da evolução do quadro durante a gestação, seguida de procedimento cirúrgico realizado precocemente por uma equipe multidisciplinar a fim de corrigir o saco herniário. ${ }^{12} \mathrm{Em}$ contraste a essa evolução, a gestação relatada não chegou até o fim, tendo o feto ido a óbito na $24^{a}$ semana de gestação.

Na situação do caso em relato, o médico obstetra, inicialmente, adotou a conduta expectante, ${ }^{13}$ todavia fez uso de medicação indutora de parto. Aproximadamente 10 horas após observar que não houve nenhum progresso no trabalho de parto, optou pela cesariana, no intuito de abreviar o sofrimento gerado pelo quadro.

\section{CONSIDERAÇÕES FINAIS}

Neste relato de caso não ficou nítida nenhuma etiologia específica para o aparecimento da encefalocele, ressaltando-se, ainda, que apesar de ter sido realizada consulta pré-concepcional com todos os exames necessários, de ter havido adequado uso de ácido fólico e sulfato ferroso e nenhuma medicação que pudesse prejudicar o desenvolvimento do feto tenha sido utilizada, o caso culminou no óbito fetal.

A presença de vários fatores etiológicos na encefalocele dificulta a confirmação de um fator causal, principalmente em casos como o descrito no presente relato, o qual mostra que, além de não terem existido causas ou fatores evidentes que possam ter levado ao desfecho ocorrido, ainda foi realizada a suplementação de ácido fólico de forma adequada/recomendada. 


\section{REFERÊNCIAS}

1. Ramos AP, Oliveira MND, Cardoso JP. Prevalência de malformações congênitas em recém-nascidos em hospital da rede pública. Rev.Saude.Com. [Internet] 2008 [citado 2017 jan 16];4(1):27-42. Disponível em: http://www.uesb.br/revista/rsc/v4/v4n1 a04.pdf

2. Horovitz DDG, Llerena JCL, Jr., Mattos RA. Atenção aos defeitos congênitos no Brasil: panorama atual. Cadernos de Saúde Pública. 2005;21 (4):1055-64. doi: 10.1590/S0102$311 \times 2005000400008$

3. Aguiar MJB, Campos AS, Aguiar RALP, Lana AMA, Magalhães RL, Babeto L T. Defeitos de fechamento do tubo neural e fatores associados em recém-nascidos vivos e natimortos. J.Pediatr. 2003;79(2):129-34. doi: 10.1590/\$0021-75572003000200007

4. Almeida LFG, Araújo JE, Crot GC, Okido MM, Berezowski AT, Duarte G et al. Epidemiological Risk Factors and Perinatal Outcomes of Congenital Anomalies. Rev. Bras. Ginecol. Obstet. 2016;38(7):348-55. doi: org/10.1055/s-0036-1586160

5. Barros ML, Campos AD, Aguiar RALP, Lana AMA, Magalhães RL, Babeto LT. Malformações do sistema nervoso central e malformações associadas diagnosticadas pela ultrassonografia obstétrica. Radiol Bras. 2012 12;45(6):309-14. doi: 10.1590/S010039842012000600005

6. Arena, JF. Encephalocele. Nord - National Organization for Rare Disorders [Internet]. 2012 [citado 2016 dez 27]. Disponível em: https://rarediseases.org/rare-diseases/encephalocele/.

7. Sharma R, Radswiki et al. Encephalocele. [Internet] [citado 2016 dez. 27]. Disponível em: https://radiopaedia.org/articles/encephalocoele

8. Paz GJ, Laborde YS, Montejo WAF. Diagnóstico ecográfico del encefalocele. MEDISAN [Internet]. 2013 [citado 2016 dez 21];17(5):871-6. Disponível em: <http://scielo.sld.cu/ pdf/san/v17n5/san18175.pdf

9. Yucetas S, Uçler N. A Retrospective Analysis of Neonatal Encephalocele Predisposing Factors and Outcomes. Pediatr Neurosurg. 2017;52:73-6. doi: 10.1159/000452805 
10. Souza LMM, Diniz CP, Souza ASR, Oliveira MAS, Noronha C, Neto. Ácido fólico na prevenção de gravidez acometida por morbidade fetal: aspectos atuais. Femina [Internet]. 2009 [citado 2018 ago 4];10;37(10):569-75. Disponível em: http://files.bvs.br/upload/S/0100-7254/2009/v37n10/a010.pdf

11. Brasil. Atenção ao pré-natal de baixo risco. Brasília; 2012.

12. Vita S. Encefalocele occipital: Reporte de un caso. RFM [Internet]. 2008 [citado 2016 dez 21 ]6;31 (1):70-4. Disponível em: http://www.scielo.org.ve/scielo.php?script=sci_arttext\&pid=\$0798-046920080001000 1 1 \&lng=es\&nrm=iso

13. Oliveira ALB, Azevedo MJA, Sanseverino MTV. Epidemiologia dos defeitos de fechamento do tubo neural no Hospital de Clinicas de Porto Alegre [monografia] [Internet]. Porto Alegre: Universidade Federal do Rio Grande do Sul; 2008. [citado 2017 jan. 26]. Disponível em: http://www.lume.ufrgs.br/handle/10183/13567

14. Siffel C, Wong LY, Olney RS, Correa A. Survival of infants diagnosed with encephalocele in Atlanta, 1979-98. Paediatr Perinat Epidemiol. [Internet] 2003 [citado 2017 jan 23];17(1):40-8. Disponível em: https://www.ncbi.nlm.nih.gov/pubmed/12562471

15. Schupp TR, Miyadahira S, Zugaib M. Qual é a conduta atual no óbito fetal? Revista da Associação Médica Brasileira. 2002;48(4):284-4. doi: 10.1590/\$0104-42302002000400017 
